\title{
SOME THEOREMS OF PHRAGMEN-LINDELOF TYPE FOR NONLINEAR PARTIAL DIFFERENTIAL EQUATIONS
}

\author{
RAMÓN QUINTANILLA
}

\begin{abstract}
The present paper studies second order partial differential equations in two independent variables of the form $\operatorname{Div}\left(\rho_{1}|u, 1|^{n-1} u, 1, \rho_{2}|u, 2|^{n-1} u, 2\right)=0$. We obtain decay estimates for the solutions in a semi-infinite strip. The results may be seen as theorems of Phragmen-Lindelof type. The method is strongly based on the ideas of Horgan and Payne [5], [6], [8].
\end{abstract}

\section{Introduction}

In [5] Horgan and Payne studied the asymptotic behaviour of solutions to the equations:

$$
\left(\rho(x, u, \nabla u) u_{, \alpha}\right)_{, \alpha}=0,
$$

on the semi-infinite strip $R=\left\{\left(x_{1}, x_{2}\right) / 0 \leq x_{2} \leq h_{1} x_{1} \geq 0\right\}$ under boundary conditions:

$$
\begin{gathered}
u\left(x_{1}, 0\right)=u\left(x_{1}, h\right)=0, \quad x_{1} \geq 0, \\
u \text { and } u, u_{a} \rightarrow 0 \text { (uniformly in } x_{2} \text { ) as } x_{1} \rightarrow \infty, \\
u\left(0, x_{2}\right)=f\left(x_{2}\right), \quad 0 \leq x_{2} \leq h,
\end{gathered}
$$

where the prescribed function $f$ is sufficiently smooth and satisfies $f(0)=$ $f(h)=0$. This study was made for two kinds of functions:

$0<m_{1} \leq \rho \leq M_{1}+K_{1} \rho\left(p^{2}+q^{2}\right)$,

(case 2)

$0<m_{2} \leq \rho^{-1} \leq M_{2}+K_{2} \rho\left(p^{2}+q^{2}\right)$, 
where $p=\partial u / \partial x_{1}$ and $q=\partial u / \partial x_{2}$. Horgan and Payne obtained exponential decay for the solutions. Recently [8] the assumption (1.2) on the bchaviour of the solution at infinity has been weakened and many others geometries for the domains have been considered.

These results were motivated by the desire to establish versions of Saint-Venant's principle in elasticity. A good survey of research on this principle in several kind of problems may be found in [3], [4]. Similar methods are currently used in the study of constrained elastic cylinders of variable cross section [11], [12]. Recent results using related methods may be found in [9], [10]. Furthermore, some considerations for the $p$-laplacian equation were given in [13] and for nonlinear fourth order equations in [15].

Alternatively, the results may be viewed as theorems of PhragmenLindelof type [2].

Following the main ideas of the work of Horgan and Payne, we generalize the results to a larger class of equations. From now on, $R$ will be a strip lying in the half plane $x_{1} \geq 0$ with fixed lateral curves $c\left(x_{1}\right), \tilde{c}\left(x_{1}\right)$ for $x_{1} \geq 0$. We may designate $R=\left\{\left(x_{1}, x_{2}\right) \in \mathbb{R}^{2} / x_{1} \geq\right.$ 0 and $\left.c\left(x_{1}\right) \leq x_{2} \leq \bar{c}\left(x_{1}\right)\right\}$. We will suppose that the width of the cross scction $L_{z}=\left\{\left(x_{1}, x_{2}\right) \in R / x_{1}=z\right\}$ is bounded above by a constant $h$. We observe that the Dirichlet homogeneous boundary condition are:

$$
u\left(x_{1}, c\left(x_{1}\right)\right)=u\left(x_{1}, \vec{c}\left(x_{1}\right)\right)=0 \text { for all } x_{1} \geq 0,
$$

and the end condition may be written as:

$$
u\left(0, x_{2}\right)=f\left(x_{2}\right) \text { for all }\left(0, x_{2}\right) \in L_{0},
$$

where the prescribed function $f$ satisfies $f(c(0))=f(\tilde{c}(0))=0$. In this paper we prove the exponential decay of classical solutions of the equation:

$$
\operatorname{Div}\left(\rho_{1}|p|^{n-1} p, \rho_{2}|q|^{n-1} q\right)=0, \quad n>0,
$$

where $\rho_{i}=\rho_{i}(x, u, \nabla u)$ in the semi-infinite strip $R$ subject to the boundary conditions (1.1b), (1.3b) and under suitable hypothesis at infinity (H.s) which is the natural translation to our case of the hypothesis used in [8]. Our results may be seen as analogues of Phragmen-Lindelof theorem for the equations and domains we study.

We establish our results for four classes of equations depending on the functions $\rho_{1}$ and $\rho_{2}$. The main result is to establish an exponential decay estimate for the energy:

$$
E_{s}(z)=\int_{R_{\pi}}|u|^{s(n+1)}\left(\rho_{1}|p|^{n+1}+\rho_{2}|q|^{n+1}\right) d A
$$


contained in the subdomain $R_{2}=\left\{\left(x_{1}, x_{2}\right) \in \mathbb{R}^{2} / 0 \leq z<x_{1}<\infty\right\} \cap R$ provided that the total energy $E_{s}(0)$ is finite for $s=0,1$. Nevertheless the method may be used for all natural numbers $s$. To ensure that the function $E_{0}$ is well defined we need to impose at infinity the condition that the solutions satisfy:

$$
\liminf _{z \rightarrow \infty} z^{-1} \int_{L_{z}}\left(\rho_{1}|p|^{n+1}+\rho_{2}|q|^{n+1}\right) d x_{2}=0
$$

while to ensure that $E_{s}(s>0)$ is well defined we suppose that (H.0) is satisfied together with the condition on the behavior of the solutions at infinity:

$$
\liminf _{z \rightarrow \infty} z^{-1} \int_{L_{z}}|u|^{s(n+1)}\left(\rho_{1}|p|^{n+1}+\rho_{2}|q|^{n+1}\right) d x_{2}<\infty .
$$

We may observe that whenever $\rho_{1}$ and $\rho_{2}$ are bounded above by a continuous function of $u, p$ and $q$ then condition (1.2) implies (H.s) for all $s \in \mathbb{N}$.

We separate the hypotheses into the following four types:

Type I

$$
\begin{aligned}
& 0<m_{1} \leq \rho_{2} \rho_{1}^{-1}, \text { and } \\
& 0<m_{2} \leq \rho_{2} \leq M_{2}+K_{2}\left(\rho_{1}|p|^{n+1}+\rho_{2}|q|^{n+1}\right) .
\end{aligned}
$$

Type II

$$
\begin{aligned}
& 0<m_{1} \leq \rho_{1}^{-1} \rho_{2}^{-1 / n} \\
& 0<\rho_{2}^{-1} \leq\left(M_{2}+K_{2}\left(\rho_{1}|p|^{n+1}+\rho_{2}|q|^{n+1}\right)\right)^{n}
\end{aligned}
$$

Type III

$$
\begin{aligned}
& 0<m_{2} \leq \rho_{1}^{n} \rho_{2}, \\
& 0<\rho_{1} \leq M_{1}+K_{1}\left(\rho_{1}|p|^{n+1}+\rho_{2}|q|^{n+1}\right) .
\end{aligned}
$$

Type IV

$$
\begin{aligned}
& 0<m_{2} \leq \rho_{2} \rho_{1}^{-n} \\
& 0<m_{1} \leq \rho_{1}^{-1} \leq M_{1}+K_{1}\left(\rho_{1}|p|^{n+1}+\rho_{2}|q|^{n+1}\right) .
\end{aligned}
$$


We observe that when $\rho_{1}=\rho_{2}$ Types I and III become identical and naturally generalize Case 1 in [5]. We can say the same thing about Types II and IV in the case $n=1$. Then our hypotheses are a natural generalization of Case 2 in [5].

A family of functions satisfying the foregoing conditions is given by:

$$
\rho_{1}=\left(1+r_{1}|p|^{n+1}+r_{2}|q|^{n+1}\right)^{y} \text { and } \rho_{2}=\left(1+r_{1}|p|^{n+1}+r_{2}|q|^{n+1}\right)^{x}
$$

where $r_{1}$ and $r_{2}$ are two positive numbers. If $0 \leq y \leq x \leq y+1$ then an easy calculation proves that these functions are of the Type I by taking $m_{1}=m_{2}=M_{2}=1$ and $K_{2}=\max \left(x r_{1}, x r_{2}\right)$. In the case $0 \leq x \leq-n y$, the functions (1.10) are of Type II by taking $m_{1}=M_{2}=1$ and $K_{2}=0$. In a similar way our functions are of Type III if $0 \leq-y \leq x / n$ by taking $m_{2}=M_{1}=1$ and $K_{1}=0$. For the Type IV we can take $m_{1}=m_{2}=M_{2}=1$ and $K_{1}=\max \left(r_{1}, r_{2}\right)$ whenever $-\frac{1}{2} \leq y \leq 0$ and $x \geq-y$.

In Section 2 we state two lemmas which are useful in the study of the exponential decay of the energies $E_{s}$ for the Type I to IV. In Section 3 we prove the exponential decay of $E_{s}$ for all the Types. We give a complete proof for Type I, but provide only a sketch for the other three types. We also give in Section 4 a theorem of decay of the energies without the restriction that the cross section is bounded above, but the hypotheses on asymptotic behavior (H.O) and (H.s) must be modified to another ( $\left.\mathrm{H}^{\prime} . \mathrm{s}\right)$. Section 5 is devoted to obtain $L^{n+1}$-estimates in the whole strip. We make a similar study in Section 6 but for the cross-section. In Section 7 we obtain an estimate for $E_{0}$ for the Neumann boundary problem. Section 8 , which concludes the paper, summarises our results by listing the various Types and measures for which exponential decay have been established. An appendix to the paper describes a method for estimating an upper bound to the total energies $E_{s}(0)$ in terms of the end conditions.

Acknowledgments. I am grateful to Prof. C. O. Horgan and Prof. R. J. Knops for their comments on an earlier version.

\section{Two previous lemmas}

In this section we establish two lemmas about integration which are useful in proving the results of Section 3. First, we generalize a weighted Poincaré inequality, due to Horgan and Payne [5, p. 314].

Lemma 2.1. Let $\rho:[0, h] \rightarrow \mathbb{R}^{+}-\{0\}$ be a continuous function and $u:[0, h] \rightarrow \mathbb{R}$ a diferentiable function such that $u(0)=u(h)=0$. Then 
there exist two constants $\beta_{r+1}$ and $B$ such that:

$$
\int_{0}^{h} \rho|u|^{r+1} d x \leq \beta_{r+1}^{-1} B^{r+1} \int_{0}^{h} \rho^{-r}|d u / d x|^{r+1} d x
$$

where $\beta_{r+1}$ depends on $r$ and $B=\int_{0}^{h} \rho d x$.

Proof: We consider the change of variable:

$$
t=\int_{0}^{x} \rho(\alpha) d \alpha
$$

so that, $d t=\rho d x$, hence we see:

$$
\int_{0}^{h} \rho|u|^{r+1} d x=\int_{0}^{B}|u|^{r+1}(t) d t .
$$

Now, we can apply Poincaré inequality $[\mathbf{1}]$, to obtain:

$$
\int_{0}^{B}|u|^{r+1} d t \leq \beta_{r+1}^{-1} B^{r+1} \int_{0}^{B}|(d u / d t)|^{r+1} d t
$$

where $\beta_{r+1}$ is a constant that depends on " $r+1$ ". Upon recalling that:

$$
d u / d t=(d u / d x)(d x / d t)=\rho^{-1} d u / d x,
$$

we obtain the desired inequality.

From Lemma 2.1 we may obtain an inequality useful in the study of the behaviour of $E_{s}$ :

$$
\begin{aligned}
& \int_{0}^{h} \rho|u|^{(r+1)(s+1)} d x \leq \\
& \quad \leq(s+1)^{r+1}\left(\beta_{r+1}\right)^{-1} B^{r+1} \int_{0}^{h} \rho^{-r}|u|^{s(r+1)}|(d u / d x)|^{r+1} d x,
\end{aligned}
$$

whenever $u(0)=u(h)=0$. This inequality may be proved by applying the former lemma to the function $u^{s+1}(s+1)^{-1}$. Now, we recall the following result which is proved in $[5$, p. 315$]$.

Lemma 2.2. Let $\rho:[0, l) \rightarrow \mathbb{R}^{+}-\{0\}$ be a continuous function such that:

$$
\int_{0}^{z} \rho d x \leq A z+B \quad \text { where } A, B \geq 0 .
$$

Then:

$$
\int_{0}^{z} \rho^{-1} d x \geq \frac{z}{A}-\frac{B}{A^{2}}, \text { for all } z>0 .
$$




\section{Decay of the energy}

In this section we state and prove some theorems for the exponential decay of the energy (1.5) for each of the types considered in Section 1. We give a complete proof only in the case of Type I. We sketch the proof in the other cases.

In order to prove the theorems, we need to introduce the function:

$$
G_{s}(z)=\frac{1}{s(n+1)+1} \int_{L_{z}} \rho_{1}|p|^{n-1} p|u|^{s(n+1)} u d x_{2} .
$$

Because of the divergence theorem, this may be rewritten as:

$$
G_{s}(z)=G_{s}\left(z_{0}\right)+\int_{z_{0}}^{z} \int_{L_{n}}|u|^{s(n+1)}\left(\rho_{1}|p|^{n+1}+\rho_{2}|q|^{n+1}\right) d A
$$

for all $z \geq z_{0} \geq 0$. Direct differentiation gives:

$$
G_{s}^{\prime}(z)=\int_{L_{z}}|u|^{s(n+1)}\left(\rho_{1}|p|^{n+1}+\rho_{2}|q|^{n+1}\right) d x_{2} .
$$

Holder's inequality applied to (3.1) leads to the inequality:

(3.4) $\left|G_{s}(z)\right| \leq$

$$
\leq \frac{1}{s(n+1)+1}\left[\int_{L_{z}} \rho_{1}|p|^{n+1}|u|^{s(n+1)} d x_{2}\right]^{\frac{n}{n+1}}\left[\int_{L_{z}} \rho_{1}|u|^{(s+1)(n+1)} d x_{2}\right]^{\frac{1}{n-3 \cdot 1}} .
$$

Now, we state the following result.

Theorem 3.1. Let $u \in C^{2}(R) \cap C^{1}(R \cup \partial R)$ be a function that satisfies equation (1.4), the boundary conditions (1.1b) and the end conditions (1.3b), (H.O) and (H.s). We suppose that $\rho_{1}$ and $\rho_{2}$ satisfy conditions of Type I(1.6). Then for all $z \geq 0$ we have:

$$
E_{5}(z) \leq E_{s}(0) e^{Q_{1}} e^{--\alpha_{1} z},
$$

where

$$
Q_{1}=\frac{K_{2} E_{0}(0)(n+1)(s(n+1)+1) m_{1}^{\frac{1}{n+2}} \beta_{n+1}^{\frac{1}{n+1}} m_{2}}{M_{2}^{2} h^{2}(s+1) n^{\frac{n}{n+1}}}
$$

and

$$
\alpha_{1}=\frac{(n+1)(s(n+1)+1) m_{1}^{\frac{1}{n+1}} \beta_{n+1}^{\frac{1}{n+1}} m_{2}}{M_{2} h(s+1) n^{\frac{n}{n+1}}} .
$$


Proof: Let $G_{s}(z)$ be defined by (3.1). On using the first inequality of (1.6) in the inequality (3.4) we obtain:

$$
\begin{aligned}
\left|G_{s}(z)\right| \leq \frac{m_{1}^{\frac{-1}{n+1}}}{s(n+1)+1}\left[\int_{L_{z}} \rho_{1}|p|^{n+1}|u|^{s(n+1)} d x_{2}\right]^{\frac{n}{n+1}} \times \\
\quad \times\left[\int_{L_{z}} \rho_{2}|u|^{(s+1)(n+1)} d x_{2}\right]^{\frac{1}{n+1}} \leq \\
\leq \frac{(s+1) B(z)}{(s(n+1)+1) m_{1}^{\frac{1}{n+1}} \beta_{n+1}^{\frac{1}{n+1}}}\left[\int_{L_{z}} \rho_{1}|p|^{n+1}|u|^{s(n+1)} d x_{2}\right]^{\frac{n}{n+1}} \times \\
\quad \times\left[\int_{L_{z}} \rho_{2}^{-n}|u|^{s(n+1)}|q|^{n+1} d x_{2}\right]^{\frac{1}{n+1}} \leq \\
\leq \frac{(s+1) n^{\frac{n}{n+1}}}{(n+1)(s(n+1)+1) m_{1}^{\frac{1}{n+1}} \beta_{n+1}^{\frac{2}{n+1}} m_{2}} B(z) \times \\
\times \int_{L_{z}}|u|^{s(n+1)}\left(\rho_{1}|p|^{n+1}+\rho_{2}\left\{\left.q\right|^{n+1}\right) d x_{2},\right.
\end{aligned}
$$

where $B(z)=\int_{L_{z}} \rho_{2}\left(z, x_{2}\right) d x_{2}$. We observe that the second inequality follows from (2.1) and the third one from the second inequality in (1.6). Thus we may conclude that:

$$
\left|G_{s}(z)\right| \leq k_{s} B(z) G_{s}^{\prime}(z)
$$

where

$$
k_{s}=\frac{(s+1) n^{\frac{n}{n+1}}}{(n+1)(s(n+1)+1) m_{1}^{\frac{1}{n+1}} \beta_{n+1}^{\frac{1}{n+1}} m_{2}},
$$

and $B(z)$ has been defined previously. From inequality (3.6) we may deduce the following two first-order differential inequalities:

$$
\begin{aligned}
G_{s}(z) & \leq k_{s} B(z) G_{s}^{\prime}(z), \\
-G_{s}(z) & \leq k_{s} B(z) G_{s}^{\prime}(z) .
\end{aligned}
$$

We now show that $G_{s}(z) \leq 0$ for all $z \geq 0$. To this end, let us suppose that there exists $z^{*} \geq 0$ such that $G_{s}\left(z^{*}\right)>0$. Then integration of (3.7) gives:

$$
G_{s}(z) \geq G_{s}\left(z^{*}\right) \times \exp \left(k_{s}^{-1} \int_{z^{*}}^{z} \frac{d \eta}{\int_{L_{\eta}} \rho_{2}\left(\eta, x_{2}\right) d x_{2}}\right) .
$$


But from (3.7) and the sccond inequality in (1.6), we have:

$$
\begin{gathered}
\int_{L_{z}}|u|^{s(n+1)}\left(\rho_{1}|p|^{n+1}+\rho_{2}|q|^{n+1}\right) d x_{2} \geq \\
\geq\left(\frac{k_{s}^{-1} G_{s}\left(z^{*}\right)}{M_{2} h+K_{2} \int_{L_{z}}\left(\rho_{1}|p|^{n+1}+\rho_{2}|q|^{n+1}\right) d x_{2}}\right) \times \\
\times \exp \left(k_{s}^{-1} \int_{z}^{z} \frac{d \eta}{M_{2} h+K_{2} \int_{L_{\eta}}\left(\rho_{1}|p|^{n+1}+\rho_{2}|q|^{n+1}\right) d x_{2}}\right),
\end{gathered}
$$

and since condition (H.0) is satisfed, we obtain:

$$
\begin{gathered}
z^{-1} \int_{L_{z}}|u|^{s(n+1)}\left(\rho_{1}|p|^{n+1}+\rho_{2}|q|^{n+1}\right) d x_{2} \geq \\
\geq \frac{k_{s}^{-1} G_{s}\left(z^{*}\right)}{z\left(M_{2} h+K_{2} \epsilon z\right)} \exp \left(\left.k_{s}^{-1} \epsilon^{-1} K_{2}^{-1} \ln \left(M_{2} h+\epsilon K_{2} \eta\right)\right|_{z} ^{z} \cdot\right)= \\
=\frac{k_{s}^{-1} G_{s}\left(z^{*}\right)}{z\left(M_{2} h+K_{2} \epsilon z\right)}\left[\frac{M_{2} h+\epsilon K_{2} z}{M_{2} h+\epsilon K_{2} z^{*}}\right] \frac{1}{\epsilon k_{2} k_{3}},
\end{gathered}
$$

for all $\epsilon$ small and for all $z \geq z(\epsilon)>0$. It then follows that:

$$
\begin{aligned}
& z^{-1} \int_{L_{z}}|u|^{s(n+1)}\left(\rho_{1}|p|^{n+1}+\rho_{2}|q|^{n+1}\right) d x_{2} \geq \\
& \quad \geq k_{s}^{-1} G_{s}\left(z^{*}\right)\left[M_{2} h+\epsilon K_{2} z\right]^{\frac{1}{k_{s} K_{2}}-2}\left[M_{2} h+\epsilon K_{2} z^{*}\right]^{\frac{-1}{\epsilon_{b_{s}} K_{2}}}
\end{aligned}
$$

On letting $z \rightarrow \infty$, we observe that for $\epsilon$ sufficiently small the right-hand side tends to infinity, which contradicts condition (H.s). Hence we have proved that $G_{s}(z) \leq 0$ for a.l $z \geq 0$. On recalling inequality (3.8), we have:

$$
E_{s}(z) \leq E_{s}(0) \exp \left(-k_{s}^{-1} \int_{0}^{z} B(\eta)^{-1} d \eta\right) .
$$

The upper bound for $\rho_{2}$ in (1.6) gives the inequality:

$$
\int_{0}^{z} \int_{L_{\eta}} \rho_{2} d A \leq M_{2} h z+K_{2} E_{0}(0),
$$

while from lemma 2.2 , we conclude:

$$
\int_{0}^{z} B(\eta)^{-1} d \eta \geq \frac{z}{M_{2} h}-\frac{K_{2} E_{0}(0)}{M_{2}^{2} h_{2}^{2}} .
$$

Thus, the desired estimate is established. 
Theorem 3.2. Let $u \in C^{2}(R) \cap C^{1}(R \cup \partial R)$ be a function that satisfes equation (1.4), the boundary conditions (1.1b) and the end conditions (1.3b), (H.O) and (H.s). We suppose that $\rho_{1}$ and $\rho_{2}$ satisfy conditions of Type II(1.7). Then for all $z \geq 0$ we have:

$$
E_{s}(z) \leq E_{s}(0) e^{Q_{2}} e^{-\alpha_{2} z},
$$

where

$$
Q_{2}=\frac{K_{2} E_{0}(0)(n+1)(s(n+1)+1) m_{1}^{\frac{1}{n+1}} \beta_{n+1}^{\frac{1}{n+1}}}{M_{2}^{2} h^{2}(s+1) n^{\frac{n}{n+1}}},
$$

and

$$
\alpha_{2}=\frac{(n+1)(s(n+1)+1) m_{1}^{\frac{3}{n+1}} \beta_{n+1}^{\frac{1}{n+1}}}{M_{2} h(s+1) n^{\frac{n}{n-1}}} .
$$

Sketch of Proof: From inequality (3.4) we obtain a similar inequality to (3.6) but with $B(z)=\int_{L_{z}} \rho_{2}^{-1 / n}\left(z, x_{2}\right) d x_{2}$ and

$$
k_{s}=\frac{(s+1) n^{\frac{n}{n+1}}}{(n+1)(s(n+1)+1) m_{1}^{\frac{1}{n+1}} \beta_{n+1}^{\frac{1}{n+1}}} .
$$

Theorem 3.3. Let $u \in C^{2}(R) \cap C^{1}(R \cup \partial R)$ be a function that satisfies equation (1.4), the boundary conditions (1.1b) and the end conditions (1.3b), (H.O) and (H.s). We suppose that $\rho_{1}$ and $\rho_{2}$ satisfy conditions of Type $H(1.8)$. Then for all $z \geq 0$ we have:

$$
E_{s}(z) \leq E_{s}(0) e^{Q_{3}} e^{-\alpha_{3} z},
$$

where

$$
Q_{3}=\frac{K_{1} E_{0}(0)(n+1)(s(n+1)+1) m_{2}^{\frac{1}{n+1}} \beta_{n+1}^{\frac{1}{n+1}}}{M_{1}^{2} h^{2}(s+1) n^{\frac{n}{n+1}}},
$$

and

$$
\alpha_{3}=\frac{(n+1)(s(n+1)+1) m_{2}^{\frac{1}{n+1}} \beta_{n+1}^{\frac{1}{n+1}}}{M_{1} h(s+1) n^{\frac{n}{n+1}}} .
$$

Sketch of Proof: As before, inequality (3.4) leads to an inequality similar to (3.6) but with $B(z)=\int_{L_{z}} \rho_{1}\left(z, x_{2}\right) d x_{2}$ and

$$
k_{s}=\frac{(s+1) n^{\frac{n}{n+1}}}{(n+1)(s(n+1)+1) m_{2}^{\frac{1}{n+1}} \beta_{n+1}^{\frac{1}{n+1}}} .
$$


Theorem 3.4. Let $u \in C^{2}(R) \cap C^{1}(R \cup \partial R)$ be a function that satisfies equation (1.4), the boundary conditions (1.1b) and the end conditions (1.3b), (H.O) and (H.s). We suppose that $\rho_{1}$ and $\rho_{2}$ satisfy conditions of Type IV(1.9). Then for all $z \geq 0$ we have:

$$
E_{s}(z) \leq E_{s}(0) e^{Q_{4}} e^{-\alpha_{4} z},
$$

where

$$
Q_{4}=\frac{K_{1} E_{0}(0)(n+1)(s(n+1)+1) m_{1}^{\frac{2}{n+1}} \beta_{n+1}^{\frac{1}{n+1}} m_{2}^{\frac{1}{n+1}}}{M_{1}^{2} h^{2}(s+1) n^{\frac{n}{n+1}}}
$$

and

$$
\alpha_{4}=\frac{(n+1)(s(n+1)+1) m_{1}^{\frac{2}{n+1}} \beta_{n+1}^{\frac{1}{n+1}} m_{2}^{\frac{1}{n+1}}}{M_{1} h(s+1) n^{\frac{n}{n+1}}} .
$$

Sketch of Proof: From inequality (3.4) we obtain an inequality as (3.6) where $B(z)=\int_{L_{z}} \rho_{1}^{-1}\left(z, x_{2}\right) d x_{2}$ and

$$
k_{s}=\frac{(s+1) n^{\frac{n}{n+1}}}{(n+1)(s(n+1)+1) m_{1}^{\frac{2}{n+1}} \beta_{n+1}^{\frac{1}{n+1}} m_{2}^{\frac{2}{n+1}}}
$$

\section{Non-bounded cross-section case}

Similar theorems may be obtained for a larger class of domains by means of the techniques used in [11], provided that the functions $\rho_{i}$ satisfy the conditions (1.6). A slight change of hypotheses permits the previous method still to be applicable. The restriction on the height of the cross section is not necessary if we change the asymptotic hypotheses (H.O) and (H.s) by:

(H's) There exists $z^{*} \geq 0$ such that:

$$
\lim _{z \rightarrow \infty} \frac{\left(\int_{L_{z}}|u|^{s(n+1)}\left(\rho_{1}|p|^{n+1}+\rho_{2}|q|^{n+1}\right) d x_{2}\right)\left(\int_{L_{x}} \rho_{2} d x_{2}\right)}{\operatorname{cxp}\left(k_{s}^{-1} \int_{z^{-}}^{z}\left(\int_{L_{\eta}} \rho_{2} d x_{2}\right)^{-1} d \eta\right)}=0
$$

Now, we suppose that $G_{s}\left(z^{*}\right)>0$ and we introduce the function:

$$
I_{s}\left(z, z^{*}\right)=\int_{z^{*}}^{z} \int_{L_{\eta}}|u|^{s(n+1)}\left(\rho_{1}|p|^{n+1}+\rho_{2}|q|^{n+1}\right) d A .
$$


From (3.7), we have:

$$
I_{s}\left(z, z^{*}\right) \geq\left(\exp k_{s}^{-1} \int_{z}^{z} \frac{d \eta}{\int_{L_{\eta}} \rho_{2}\left(\eta, x_{2}\right) d x_{2}}-1\right) G_{s}\left(z^{*}\right) .
$$

On assuming that:

$$
\int_{z^{*}}^{\infty} \frac{d \eta}{\int_{L_{\eta}} \rho_{2} d x_{2}}=\infty
$$

we may apply the l'Hopital Rule to (4.1) to obtain the inequality:

$$
\frac{\left(\int_{L_{z}}|u|^{s(n+1)}\left(\rho_{1}|p|^{n+1}+\rho_{2}|q|^{n+1}\right) d x_{2}\right)\left(\int_{L_{z}} \rho_{2} d x_{2}\right)}{\exp \left(k_{s}^{-1} \int_{z^{*}}^{z}\left(\int_{L_{\eta}} \rho_{2} d x_{2}\right)^{-1} d \eta\right)} \geq k_{s} G_{s}\left(z^{*}\right) \text {. }
$$

Thus, when $G_{s}\left(z^{*}\right)>0$ (4.1) can not hold simultaneously with the asymptotic condition (H's) and we conclude that $G_{s}(z) \leq 0$ for all $z \geq 0$. Hence we may establish the estimate:

$$
E_{s}(z) \leq E_{s}(0) \exp \left(-k_{s}^{-1} \int_{0}^{z} \frac{d \eta}{\int_{L_{\eta}} \rho_{2} \overline{d x_{2}}}\right)
$$

Thus, we have :

Theorem 4.1. Let $u \in C^{2}(R) \cap C^{1}(R \cup \partial R)$ be a function that satisfies equation (1.4), the boundary conditions (1.1b) and the end conditions (1.3b), ( $\left.H^{\prime}, s\right)$ and (4.2). We suppose that $\rho_{1}$ and $\rho_{2}$ satisfy conditions of Type I(1.6). Then for all $z \geq 0$ we have:

$$
E_{s}(z) \leq E_{s}(0) \exp \left(\frac{E_{0}(0) k_{s}^{-1} K_{2}}{M_{2}^{2} l_{\alpha}^{2}}\right) \exp \left(-\frac{k_{s}^{-1}}{M_{2}} \int_{0}^{z} \frac{d \eta}{l(\eta)}\right)
$$

where $k_{s}$ is given in the proof of Theorem 3.1,l( $\left.\eta\right)$ is the height of the cross-section $L_{\eta}$ and $l_{\alpha}=\inf _{\eta \in[0, \infty)} l(\eta)$.

Of course, Theorem 3.1 is related with Theorem 4.1. Futhermore, if we suppose that $l(\eta) \leq C \eta$ and $\frac{k_{0}^{-1}}{M_{2} C}>2$, then

$$
\lim _{z \rightarrow \infty} z^{-1} \int_{L_{x}}\left(\rho_{1}|p|^{n+1}+\rho_{2}|q|^{n+1}\right) d x_{2}=0
$$

and

$$
\lim _{z \rightarrow \infty} z^{-1} \int_{L_{z}}|u|^{s(n+1)}\left(\rho_{1}|p|^{n+1}+\rho_{2}|q|^{n+1}\right) d x_{2}<\infty .
$$

are sufficient conditions to garantee the decay of solutions.

We also may obtain some theorems for the Types II, III, IV by suitable modifying the function $\rho_{2}$ by $\rho_{2}^{-1 / n}, \rho_{1}$ and $\rho_{1}^{-1}$ in conditions (4.2) and $\left(\mathrm{H}^{\prime}, \mathrm{s}\right)$. 


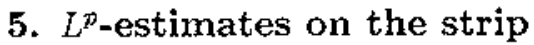

The aim of this section is to obtain $L^{p}$-estimates for the solutions of our equations for the Types I to IV. To this end we use the estimates for the energies $E_{s}$ obtained in the Section 3. Thus, our object is to examine the asymptotic behaviour of the function:

$$
J(z)=\int_{R_{z}}|u|^{n+1} d A
$$

Theorem 5.1. Let $u \in C^{2}(R) \cap C^{1}(R \cup \partial R)$ be a function that satisfies $(1.1 b),(1.3 b),(1.4)$ and $E_{0}(0)<\infty$. We suppose that $0<d_{2} \leq \rho_{2}$. Then for all $z \geq 0$ we have:

$$
J(z)=\int_{R_{z}}|u|^{n+1} d A \leq\left(d_{2} \beta_{n+1}\right)^{-1} h^{n+1} E_{0}(z) .
$$

Proof: First, we may recall the Poincaré inequality:

$$
\int_{L_{z}}|u|^{n+1} d x_{2} \leq \beta_{n+1}^{-1} h^{n+1} \int_{L_{z}}|q|^{n+1} d x_{2}
$$

which on using the hypothesis on $\rho_{2}$ enables us to deduce that

$$
\int_{L_{z}}|u|^{n+1} d x_{2} \leq\left(d_{2} \beta_{n+1}\right)^{-1} h^{n+1} \int_{L_{z}} \rho_{2}|q|^{n+1} d x_{2} .
$$

Now, if we integrate the former inequality from $z$ to infinity we obtain:

$$
\begin{aligned}
J(z)=\int_{R_{z}}|u|^{n+1} d A & \leq\left(d_{2} \beta_{n+1}\right)^{-1} h^{n+1} \int_{R_{z}} \rho_{2}|q|^{n+1} d A \leq \\
& \leq\left(d_{2} \beta_{n+1}\right)^{-1} h^{n+1} E_{0}(z) .
\end{aligned}
$$

Remark 5.1. The assumption on $\rho_{2}$ is automatically satisfied for the functions of Type I. Then, we conclude the estimate for the solutions in this case. Moreover, we also can derive a similar estimate for the solutions of the farnily given in (1.10) whenever $0 \leq x$.

Theorem 5.2. Let $u \in C^{2}(R) \cap C^{1}(R \cup \partial R)$ be a function that satisfies (1.1b), (1.3b), (1.4), $E_{0}(0)<\infty$ and $E_{1}(0)<\infty$. We also suppose that $n$ is a natural number and $0<\rho_{2}^{-1} \leq\left(D_{2}+F_{2}\left(\rho_{1}|p|^{n+1}+\rho_{2}|q|^{n+1}\right)\right)^{n}$. 
Then, there exist " $n+1$ " positive numbers $k_{i}, 0 \leq i \leq n$ such that for all $z \geq 0$ we have:

$$
\begin{aligned}
J(z)= & \int_{R_{z}}|u|^{n+1} d A \leq \\
& \leq\left(\left(\sum_{1 \leq i \leq n} k_{i} E_{1}^{i}(z) E_{0}(z)\right)^{\frac{n+1}{i+1}}+k_{0} E_{1}^{n}(z) E_{0}(z)\right)^{\frac{1}{n+1}} .
\end{aligned}
$$

Proof: Let us consider :

$$
J(z)=\int_{R_{z}}|u|^{n+1} d A=-(n+1) \int_{R_{z}} \epsilon(u)\left(x_{2}-c\left(x_{1}\right)\right)|u|^{n} q d A,
$$

where $\epsilon(u)$ is the sign of $u$. On using the Holder inequality with the hypothesis on $\rho_{2}$ we find:

$$
\begin{aligned}
J(z) & \leq h(n+1)\left(\int_{R_{z}} \rho_{2}^{-1 / n}|u|^{n+1} d A\right)^{\frac{n}{n+1}}\left(\int_{R_{z}} \rho_{2}|q|^{n+1} d A\right)^{\frac{1}{n+1}} \\
& \leq h(n+1)\left(D_{2} J(z)+F_{2} E_{1}(z)\right)^{\frac{n}{n+1}} E_{0}(z)^{\frac{1}{n+1}}
\end{aligned}
$$

and hence, we conclude:

$$
J(z)^{n+1} \leq(h(n+1))^{n+1}\left(D_{2} J(z)+F_{2} E_{1}(z)\right)^{n} E_{0}(z) .
$$

Using now the Newton formulae we see:

$$
\begin{aligned}
J(z)^{n+1} \leq\left(\sum_{1 \leq i \leq n}\left(\frac{J(z)}{2 n}\right)^{n-i}(2 n)^{n-i}\left(\begin{array}{c}
n \\
i
\end{array}\right)\left(\frac{F_{2}}{D_{2}}\right)^{i} E_{1}^{i}(z)+\right. \\
\left.+\left(\frac{F_{2}}{D_{2}}\right)^{n} E_{1}^{n}(z)\right)(h(n+1))^{n+1} D_{2}^{n} E_{0}(z) .
\end{aligned}
$$

But we have the inequality $a b \leq \frac{a^{p}}{p}+\frac{b^{q}}{q}$, whenever $p^{-1}+q^{-1}=1$, and so we may obtain:

$$
\begin{gathered}
J(z)^{n+1} \leq 2\left(\sum_{1 \leq i \leq n}\left((2 n)^{n-i}\left(\begin{array}{l}
n \\
i
\end{array}\right)\left(\frac{F_{2}}{D_{2}}\right)^{i}(h(n+1))^{n+1} D_{2}^{n} E_{1}^{i}(z) E_{0}(z)\right)^{\frac{n+1}{i+1}}+\right. \\
\left.+(h(n+1))^{n+1}{F_{2}}^{n} E_{1}^{n}(z) E_{0}(z)\right)
\end{gathered}
$$

which leads to the desired inequality. 
Remark 5.2. In a similar way to that noted in the remark at the end of Theorem (5.1), the hypothesis imposed on $\rho_{2}$ is automatically satisfied for the Type II. An easy calculation shows that if $\rho_{1}$ and $\rho_{2}$ are of Type III or IV, then we have the inequality $0<\rho_{2}^{-1} \leq\left(m_{2}^{-1 / n} M_{1}+\right.$ $\left.m_{2}^{-1 / n} K_{1}\left(\rho_{1}|p|^{n+1}+\rho_{2}|q|^{n+1}\right)\right)^{n}$. Of course the hypothesis on $\rho_{2}$ is automaticaly satisfied for the functions of Type $I$. Then, we obtain estimates of the solutions for all four types.

Remark 5.3. We may generalize the previous results for the case that $n \notin \mathbb{N}$. To this end we should make a manipulation from (5.3) that allows to obtain the inequality

$$
J(z)^{I(n+1)+1} \leq(h(n+1))^{n+1}\left(D_{2} J(z)+F_{2} E_{1}(z)\right)^{I(n+1)} E_{0}(z),
$$

where $I(n+1)$ is the maximum integer which is less than $n+1$. Now, we may obtain an estimate for $J(z)$ is a similar way to the proof of Theorem 5.2 .

\section{6. $L^{p}$-estimates on the cross-section}

In this section, we obtain $L^{p}$-estimates on the cross-section for the solutions of our equations. Under suitable hypotheses, we establish the exponential decay of the function:

$$
H(z)=\int_{L_{z}}|u|^{n+1}\left(z, x_{2}\right) d x_{2} .
$$

Theorem 6.1. Let $u \in C^{2}(R) \cap C^{l}(R \cup \partial R)$ be a function that satisfies (1.1b), (1.3b) and (1.4). We suppose that $0<d_{1} \leq \rho_{1}, J(0)<\infty$ and $E_{0}(0)<\infty$. Then for all $z \geq 0$ we have:

$$
H(z)=\int_{L_{z}}|u|^{n+1} d x_{2} \leq(n+1) d_{1}^{-1 /(n+1)} E_{0}(z)^{1 /(n+1)} J(z)^{n /(n+1)} .
$$

Proof: First, we observe

$$
\begin{aligned}
\int_{L_{z}}|u|^{n+1} d x_{2}= & -(n+1) \int_{R_{z}} \epsilon(u)|u|^{n} p d A \leq \\
& \leq(n+1)\left(\int_{R_{z}}|u|^{n+1} d A\right)^{\frac{n}{n+1}}\left(\int_{R_{z}}|p|^{n+1} d A\right)^{\frac{1}{n+1}} .
\end{aligned}
$$

Using the hypothesis on $\rho_{1}$, we find:

$$
\begin{aligned}
H(z) & \leq d_{1}^{-1 /(n+1)}(n+1)\left(\int_{R_{z}}|u|^{n+1} d A\right)^{\frac{n}{n+1}}\left(\int_{R_{z}} \rho_{1}|p|^{n+1} d A\right)^{\frac{1}{n+1}} \leq \\
& \leq d_{1}^{-1 /(n+1)}(n+1) E_{0}(z)^{1 /(n+1)} J(z)^{n /(n+1)} \text {. }
\end{aligned}
$$


Remark 6.1. The hypothesis on $\rho_{1}$ is satisfied for the elements of the family $(1.10)$ where $0 \leq y$. If we suppose that $\rho_{1}=\rho_{2}$ the hypothesis on $\rho_{1}$ is automatically satisfied for functions of Type I and III.

Theorem 6.2. Let $u \in C^{2}(R) \cap C^{1}(R \cup \partial R)$ be a function that satisfies $(1.1 b),(1.3 b)$ and (1.4). We suppose that $0<\rho_{1}^{-1} \leq\left(D_{1}+F_{1}\left(\rho_{1} \mid p\right)^{n+1}+\right.$ $\left.\left.\rho_{2}|q|^{n+1}\right)\right)^{n}, E_{0}(0)<\infty, E_{1}(0)<\infty$ and $J(0)<\infty$. Then for all $z \geq 0$ we have:

$H(z)=\int_{L_{z}}|u|^{n+1} d x_{2} \leq(n+1) E_{0}(z)^{1 /(n+1)}\left(D_{1} J(z)+F_{1} E_{1}(z)\right)^{n /(n+1)}$.

Proof: First, we observe:

$$
\begin{aligned}
H(z)= & -(n+1) \int_{R_{z}} \epsilon(u)|u|^{n} p d A \leq \\
& \leq(n+1)\left(\int_{R_{z}} \rho_{1}^{-1 / n}|u|^{n+1} d A\right)^{\frac{n}{n+1}}\left(\int_{R_{z}} \rho_{1}|p|^{n+1} d A\right)^{\frac{1}{n+1}} .
\end{aligned}
$$

Then from the the hypothesis on $\rho_{1}$, we obtain:

$$
H(z) \leq(n+1) E_{0}(z)^{1 /(n+1)}\left(D_{1} J(z)+F_{1} E_{1}(z)\right)^{n /(n+1)} .
$$

Remark 6.2. For $n \geq 1$ and $0<\rho_{1}^{-1} \leq\left(D_{1}+F_{1}\left(\rho_{1}|p|^{n+1}+\rho_{2}|q|^{n+1}\right)\right)$, then $0<\rho_{1}^{-1} \leq\left(D_{1}^{\prime}+F_{1}\left(\rho_{1}|p|^{n+1}+\rho_{2}|q|^{n+1}\right)\right)^{n}$ where $D_{1}^{\prime}=\max \left(1, D_{1}\right)$. Thus, we may derive an estimate whenever $\rho_{1}$ and $\rho_{2}$ are of Type IV. If we suppose that $\rho_{1}=\rho_{2}$ the hypothesis on $\rho_{1}$ is automatically satisfied for the functions of Types I to IV. 61])

Remark 6.3. By recalling the Sobolev type inequality (see [14, p.

$$
\left(\sup _{x \in[0, h]}|u(x)|\right)^{p} \leq K^{p} h^{p-1} \mid\|u\|_{W^{1, D}}^{p},
$$

we may also conclude decay estimates for the function

$$
L(z)=\max _{x_{2} \in L_{z}}\left|u\left(z, x_{2}\right)\right|,
$$

when the cross sections are bounded.

\section{Neumann boundary condition}

Until now, our results on solutions of the equations (1.4) have been subject to homogeneous Dirichlet boundary conditions. The aim of this 
section is to sketch similar results for Neumann conditions, whenever the cross- section is the interval $[0, \mathrm{~h}]$. A deep study of this problem for the case $\rho_{1}=\rho_{2}$ and $n=1$ may be found in [10]. Thus we examine the equations (1.4) subject to the conditions at infinity (H.0), and the end condition:

$$
\rho_{1}|p|^{n-1} p=g\left(x_{2}\right) \text { on } x_{1}=0,
$$

and the boundary conditions:

$$
q\left(x_{1}, 0\right)=q\left(x_{1}, l\right)=0 \text { for all } x_{1} \geq 0
$$

We also make an assumption on the end condition (1.3) which is related to the condition that the load be self-equilibrated for an elastic material (see among others [3], [7]):

$$
\int_{L_{0}} g\left(x_{2}\right) d x_{2}=0
$$

On using the divergence theorem, we see that the former assumption implies:

$$
\int_{L_{z}} \rho_{1}|p|^{n-1} p d x_{2}=0 \text { for all } x_{l} \geq 0
$$

Now, we take $v\left(x_{1}, x_{2}\right)=u\left(x_{1}, x_{2}\right)-\tilde{u}\left(x_{1}\right)$, where $\tilde{u}$ will be explicitly defined later. We remark that $q(u)=q(v)$. Because of (7.2) we have:

$$
E_{0}(z)=-\int_{L_{z}} \rho_{1}|p|^{n-1} p v d x_{2}
$$

Direct differentiation of (1.5), with $s=0$, yields:

$$
E_{0}^{\prime}(z)=-\int_{L_{z}}\left(\rho_{1}|p|^{n+1}+\rho_{2}|q|^{n+1}\right) d x_{2}
$$

In a similar way to the derivation of (3.11), we obtain the following inequality for $E_{0}$ :

(7.5) $E_{0}^{\prime}(z)+\alpha(z) E_{0}(z) \leq-\int_{L_{z}} \rho_{2}|q|^{n+1} d x_{2}+\alpha^{n+1}(z) \int_{L_{z}} \rho_{1} v^{n+1} d x_{2}$. 
Now, we may obtain similar estimates to (3.5), (3.12), (3.13) and (3.14) with $s=0$ by taking respectively:

$$
\begin{aligned}
& \tilde{u}\left(x_{1}\right)=\frac{\int_{L_{z}} u \rho_{2} d x_{2}}{\int_{L_{z}} \rho_{2} d x_{2}} \text { for the Type I, } \\
& \tilde{u}\left(x_{1}\right)=\frac{\int_{L_{z}} u \rho_{2}^{-1 / n} d x_{2}}{\int_{L_{z}} \rho_{2}^{-1 / n} d x_{2}} \text { for the Type II, } \\
& \tilde{u}\left(x_{1}\right)=\frac{\int_{L_{z}} u \rho_{1} d x_{2}}{\int_{L_{z}} \rho_{1} d x_{2}} \text { for the Typc III, } \\
& \tilde{u}\left(x_{1}\right)=\frac{\int_{L_{z}} u \rho_{1}^{-1} d x_{2}}{\int_{L_{z}} \rho_{1}^{-1} d x_{2}} \text { for the Type IV. }
\end{aligned}
$$

\section{Summary}

To conclude we summarize briefly the cases where we have established exponential decay.

In Section 3, we have proved the exponential decay of the energies $E_{s}$ for all the Types (I to IV) whenever (H.0) and (H.s) are satisfied and the cross-section is bounded. In Section 5, we have proved that if (H.O) is satisfied and the cross section is bounded, then there is exponential decay of $J(z)$ for the functions of Type I. We may say the same thing for many functions of Type III. We have also established the exponential decay of $J(z)$ for solutions of the equations containing the functions of Type II, III and IV whenever (H.0) and (H.s) are satisficd and the cross-section is bounded.

In Section 6, we have proved that if (H.0) is satisfied and the crosssection is bounded, we may conclude the exponential decay of $H(z)$ for functions of Type I. We have also proved the exponential decay of $H(z)$ for the solutions of the equations having functions of Type IV whenever (H.O) and (H.s) are satisfied and the cross-section is bounded. Some comments on the behavior of the function $L(z)$ are also stated.

In order to obtain a good estimate of the decay for $J(z)$ and $H(z)$ we may use inequalities (5.1), (5.2), (6.1) and (6.2). Note however that we have not found estimates for the values of the $k_{i}$ in (5.2) nor when we estimate $H(z)$ in (6.2). Nevertheless, it should be possible to obtain these values.

In Section 4, we have extended the exponential decay results for the energy to an enlarged class of domains. 
Section 7 is devoted to obtaining exponential decay of $E_{0}$ for the Neumann problem, whenever the cross section is constant, for the 'Types I to IV.

\section{Appendix A: Total energy bounds}

The total energies:

$$
E_{s}(0)=\int_{R}|u|^{s(n+1)}\left(\rho_{1}|p|^{n+1}+\rho_{2}|q|^{n+1}\right) d A
$$

contained in the strip appears in the estimates. We now obtain upper bounds for $E_{s}(0)$ in terms of the end data for functions of Type I. Similar estimates may be obtained for the other types. We will consider that $R$ is determined by two straight lines:

$$
c\left(x_{1}\right)=c_{1} x_{1} \text { and } \vec{c}\left(x_{1}\right)=c_{2} x_{1}+h .
$$

For an arbitrary $C^{1}$ function $\phi\left(x_{1}, x_{2}\right)$, defined on $R$, Holder's inequality yields:

$$
\begin{aligned}
& \int_{R}\left(\rho_{1}|p|^{n-1} p \phi_{, 1}+\rho_{2}|q|^{n-1} q \phi_{, 2}\right)|u|^{s n} \phi^{s} d A \leq \\
& \quad \leq E_{s}(0)^{\frac{n}{n+1}}\left(\int_{R}\left(\rho_{1}|\phi, 1|^{n+1}+\rho_{2}|\phi, 2|^{n+1}\right)|\phi|^{s(n+1)} d A\right)^{\frac{1}{n+1}} .
\end{aligned}
$$

If $\phi$ is chosen to satisfy (1.1b), (1.2) and (1.3), the divergence theorem shows that the left hand term of (A.2) is:

$$
\frac{-1}{s(n+1)+1} \int_{L_{0}} \epsilon(u) \rho_{1}|p|^{n-1} p|u|^{s(n+1)} u d x_{2}=E_{s}(0) \text {. }
$$

Thus, we obtain:

$$
E_{s}(0) \leq \int_{R}\left(\rho_{1}|\phi, 1|^{n+1}+\rho_{2}|\phi, 2|^{n+1}\right)|\phi|^{s(n+1)} d A .
$$

For the functions of Type I we have from (A.4):

$$
\begin{gathered}
E_{s}(0) \leq \int_{R} \rho_{2}\left(m_{1}^{-1}|\phi, 1|^{n+1}+|\phi, 2|^{n+1}\right)|\phi|^{s(n+1)} d A \leq \\
\leq \int_{R}\left(M_{2}+K_{2}\left(\rho_{1} p^{n+1}+\rho_{2} q^{n+1}\right)\right)\left(m_{1}^{-1}|\phi, 1|^{n+1}+|\phi, 2|^{n+1}\right)|\phi|^{s(n+1)} d A \leq \\
\leq M_{2} \int_{R}\left(m_{1}^{-1}|\phi, 1|^{n+1}+|\phi, 2|^{n+1}\right)|\phi|^{s(n+1)} d A+ \\
\text { (A.5) } \quad+K_{2} \max _{R}\left(m_{1}^{-1}|\phi, 1|^{n+1}+|\phi, 2|^{n+1}\right)|\phi|^{s(n+1)} E_{0}(0) .
\end{gathered}
$$


Thus, on setting $\mathcal{F}(\phi)=1-K_{2} \max _{R}\left(m_{1}^{-1}|\phi, 1|^{n+1}+|\phi, 2|^{n+1}\right)>0$, we obtain :

$$
E_{0}(0) \leq \mathcal{F}(\phi)^{-1} M_{2} \int_{R}\left(m_{1}^{-1}|\phi, 1|^{n+1}+|\phi, 2|^{n+1}\right) d A
$$

Let us take $\phi=f\left(\frac{\left(x_{2}-c_{1} x_{1}\right) h}{\left(c_{2}-c_{1}\right) x_{1}+h}\right) e^{-\gamma x_{1}}$. Easy calculations lead to:

$$
|\phi, 1| \leq e^{-\gamma x_{1}}\left[\gamma|f|+\left|f^{\prime}\right| \frac{c_{2} h}{\left(c_{2}-c_{1}\right) x_{1}+h}\right]
$$

and

$$
\phi, 2=e^{-\gamma x_{1}} f^{\prime}\left(\frac{\left(x_{2}-c_{1} x_{1}\right) h}{\left(c_{2}-c_{1}\right) x_{1}+h}\right) \frac{h}{\left(c_{2}-c_{1}\right) x_{1}+h} .
$$

On supposing that

$$
K_{2}^{-1}>\max _{[0 ; h]}\left(\left|f^{\prime}\left(x_{2}\right)\right|^{n+1}+m_{1}^{-1}\left[\gamma\left|f\left(x_{2}\right)\right|+c_{2}\left|f^{\prime}\left(x_{2}\right)\right|\right]^{n+1}\right),
$$

we have an upper bound: $E_{0}(0) \leq$

$\leq \frac{M_{2} \int_{R} e^{-(n+1) \gamma x_{1}}\left(m_{1}^{-1}\left[\gamma|f|+\left|f^{\prime}\right| \frac{c_{3} h}{\left(\left\{c_{1}-c_{1}\right) x_{2}+h\right.}\right]^{n+1}+\left|f^{\prime}\right|^{n+1}\left(\frac{h}{\left(c_{2}-c_{1}\right) x_{1}+h}\right)^{n+1} d A\right.}{1-K_{2} \max _{\mid 0, h]}\left(\left|f^{\prime}\left(x_{2}\right)\right|^{n+1}+m_{1}^{-1}\left[\gamma\left|f\left(x_{2}\right)\right|+\left.c_{2}\left|f^{\prime}\left(x_{2}\right)\right|\right|^{n+1}\right)\right.}$.

Thus:

$$
\begin{aligned}
& E_{0}(0) \leq \\
& \leq \frac{\frac{M_{2}}{h}\left[\frac{h}{(n+1) \gamma}+\frac{c_{2}-c_{1}}{(n+1)^{2} \gamma^{2}}\right]\left(\int_{0}^{h}\left(m_{1}^{-1}\left[\gamma|f|+c_{2}\left|f^{\prime}\right|\right]^{n+1}+\left|f^{\prime}\right|{ }^{n+1}\right) d x_{2}\right)}{1-K_{2} \max _{[0, h]}\left(\left|f^{\prime}\left(x_{2}\right)\right|^{n+1}+m_{1}^{-1}\left[\gamma\left|f\left(x_{2}\right)\right|+c_{2}\left|f^{\prime}\left(x_{2}\right)\right|\right]^{n+1}\right)}
\end{aligned}
$$

which after inscrtion into inequality (A.5), yields the estimate:

$$
\begin{gathered}
E_{s}(0) \leq K_{2} \max _{[0, h]} f^{s(n+1)}\left(\left|f^{\prime}\left(x_{2}\right)\right|^{n+1}+\right. \\
\left.+m_{1}^{-1}\left[\gamma\left|f\left(x_{2}\right)\right|+c_{2}\left|f^{\prime}\left(x_{2}\right)\right|\right]^{n+1}\right) E_{0}(0)+ \\
+\frac{M_{2}}{h}\left[\frac{h(n+1) \gamma(1+s(n+1))+\left(c_{2}-c_{1}\right)}{(n+1)^{2} \gamma^{2}(1+s(n+1))^{2}}\right] \times \\
\left.\times\left(\int_{0}^{h}|f|^{s(n+1)}\left(m_{1}^{-1}|\gamma| f\left|+c_{2}\right| f^{\prime} \mid\right]^{n+1}+\left|f^{\prime}\right|^{n+1}\right) d x_{2}\right) .
\end{gathered}
$$




\section{References}

1. H. BRezIs, "Analyse fonctionnelle," Masson, Paris, 1983.

2. J. B. Conway, "Functions of one complex variable," G.T.M., Springer-Verlag, New York, Second Edition, 1978.

3. C. O. HORGAN AND J. K. KNowles, "Recent developments concerning Saint-Venant's Principle," Advances in Applied Mechanics 23, (Ed. J. W. Hutchinson and T. Y. Wu), Academic Press, New York, 1983, pp. 179-269.

4. C. O. Horgan, Recent developments concerning Saint-Venant's Principle: An update, Appl. Mechanics Reviews 42 (1989), 295-303.

5. C. O. Horgan and L. E. PAYNe, Decay estimates for second-order quasilinear partial differential equations, Adv. in Applied Math. 5 (1984), 309-332.

6. C. O. Horgan AND L. E. PAyne, Decay estimates for a class of second-order quasilinear equations in three dimensions, Arch. Rat. Mech. Anol. 86 (1984), 279-289.

7. R. J. Knops and L. E. PAyne, A Saint-Venant Principle for nonlinear elasticity, Arch. Rat. Mech. Anal. 81 (1983), 1-12.

8. C. O. Horgan ANd L. E. PAYNE, Decay Estimates for a class of nonlinear boundary value problems in two dimensions, S.I.A.M. Jour. Math. Anal. 20 (1989), 782-788.

9. C. O. HORGAN AND L. E. PAYNe, On the asymptotic behavior of solution of inhomogeneous second-order quasilinear partial differential equations, Quat. Appl. Mathematics XLVII (1989), 753-771.

10. C. O. Horgan and L. E. Payne, On Saint-Venant's Principle in finite anti-plane shear: An energy approach, Arch. Rat. Mech. Anal. 109 (1990), 107-137.

11. J. N. Flavin, R. J. Knops and L. E. Payne, Decay estimates for constrained elastic cylinder of variable cross section, Quat. Appl. Mathematics XLVII (1989), 325-350.

12. R. J. KNops, S. RIONERo aNd L. E. PAYNe, Saint- Venant's principle on unbounded regions, Proc. Roy. Soc. Edinburgh 115A (1990), 319-336.

13. J. N. Flavin, R. J. Knops and L. E. Payne, Asymptotic behavior of solution to semi-linear elliptic equations on the half-cylinder, Jour. Appl. Math. Phys.(ZAMP) 43 (1992), 405-421. 
14. V. G. MAZ'JA, "Sobolev spaces," Springer-Verlag, Berlin, 1985.

15. C. O. Horgan AND L. E. PAYNE, A Saint- Venant principle for a theory of nonlinear plane clasticity, Quat. Appl. Mathematics L (1992), 641-675.

Departament de Matemàtica Aplicada. II

Universitat Politècnica de Catalunya.

Terrassa, Barcelona.

SPAIN

Rebut el 2 de Juliol de 1993 